Quantum phases: 50 years of the Aharonov-Bohm Effect and 25 years of the Berry phase

\title{
Investigation of the coupling asymmetries at double-slit interference experiments
}

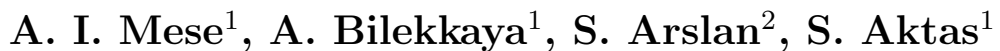 \\ and \\ A. Siddiki $\mathbf{i}^{2,3}$ \\ 1 Trakya University, Department of Physics, 22030 Edirne, Turkey \\ ${ }^{2}$ Department of Physics, Arnold Sommerfeld Center for Theoretical Physics, \\ Ludwig-Maximilans-Universitat, Theresienstrasse 37, 80333 Munich, Germany \\ ${ }^{3}$ Istanbul University, Faculty of Sciences, Physics Department, Vezneciler-Istanbul \\ 34134, Turkey
}

\begin{abstract}
Double-slit experiments inferring the phase and the amplitude of the transmission coefficient performed at quantum dots (QD), in the Coulomb blockade regime, present anomalies at the phase changes depending on the number of electrons confined. This phase change cannot be explained if one neglects the electron-electron interactions. Here, we present our numerical results, which simulate the real sample geometry by solving the Poisson equation in $3 \mathrm{D}$. The screened potential profile is used to obtain energy eigenstates and eigenvalues of the QD. We find that, certain energy levels are coupled to the leads stronger compared to others. Our results give strong support to the phenomenological models in the literature describing the charging of a QD and the abrupt phase changes.
\end{abstract}

\section{Introduction}

One of the most interesting experiments in the history of physics is the double-slit experiments, which infers to the quantum mechanical nature of the particles. The technological developments in producing low dimensional high mobility charge carrier systems, enabled experimentalists to re-do the double-slit experiments considering nanostructures. In the experiments performed at cryogenic temperatures and considering a two dimensional electron system (2DES), the phase and the transmission amplitude were measured simultaneously [1, 2]. The findings of these and consequent experiments activated a huge number of theoreticians to understand the physics underlying the abrupt phase changes [3, 4, 5, 6, 7, 8], for a comprehensive review we suggest the reader to check especially Ref. [5] and the references given thereby. In particular, G. Hackenbroich et. al investigated the effect of shape deformation of a parabolic quantum dot (QD), in the absence of Coulomb interaction, and showed that the degeneracy due to the symmetry of the QD is lifted, however, for the deformed QD 
it is still possible to obtain density of states in broad energy intervals, which are large compared to the single particle level spacings $\delta$. The interplay between the level width $\Gamma$ and $\delta$ is used to give an explanation to the observed phase anomalies [6, 8]. On the other hand, the effect of interactions was included by M. Stopa by solving the related Poisson and Schrödinger equations self-consistently within a Hartree-Fock type mean field approximation, however, its influence on the phase was left unresolved. We should also note that, in these calculations a rather simplified QD geometry was investigated compared to the experiments.

This work aims to provide numerical support to the theories which rely on the formation of a wide state at certain QD geometries. We obtain the potential profile of the real samples by solving the Poisson equation in 3D using fast Fourier transformation, iteratively [9, 10]. In our calculations, we consider the sample geometry presented in Ref. [2. The next step is to obtain the energy eigenstates and values for the calculated effective potential. We solve the Schrödinger equation by diagonalizing the singleparticle Hamiltonian implementing the finite difference techniques.

\section{Theory}

Here, we investigate the single particle eigen-energies and eigenfunctions of the reduced 2D Hamiltonian

$$
H=\frac{\mathbf{p}^{2}}{2 m^{*}}+V(x, y)
$$

where $\mathbf{p}$ is the momentum operator in $2 \mathrm{D}, m^{*}$ is the effective mass $\left(=0.067 m_{e}\right.$ in GaAs) and $V(x, y)=V_{G}(x, y)+V_{D}(x, y)+V_{l}(x, y)+V_{H}(x, y)$ is the mean field potential composed of gates, leads, donors and Hartree terms, respectively.

In physics, WKB approximation is one of the most frequently applied approximation to solve Schrdinger's equation. The transmission amplitude $W_{n}(a, b)$ is calculated at the barrier along the classical turning points $(\mathrm{a}, \mathrm{b})$, via

$$
\begin{aligned}
& W_{n}(a, b)(E)=\frac{e^{\xi(E)}}{1+\frac{1}{4} e^{\xi(E)}}, \\
& \xi(E)=-2 \int_{a}^{b} d x \sqrt{\frac{2 m}{\hbar^{2}}(V(x)-E)} .
\end{aligned}
$$

It is known that the transmission amplitude depend almost linearly to the energy of the incoming state, assuming plane waves and within the WKB approximation [11], which we utilize likewise in the following to calculate transport through the QD.

We proceed our work by considering the real geometry and the potential profile calculated within the self-consistent Thomas-Fermi-Poisson (TFP) theory. In the following section we first discuss the limitations of such a mean field approximation and compare our method with the existing calculation schemes in the high electron occupation regime, i.e. $N \gtrsim 100$. We show that, the single particle energy states and energies can be well described in this regime considering TFP theory. 


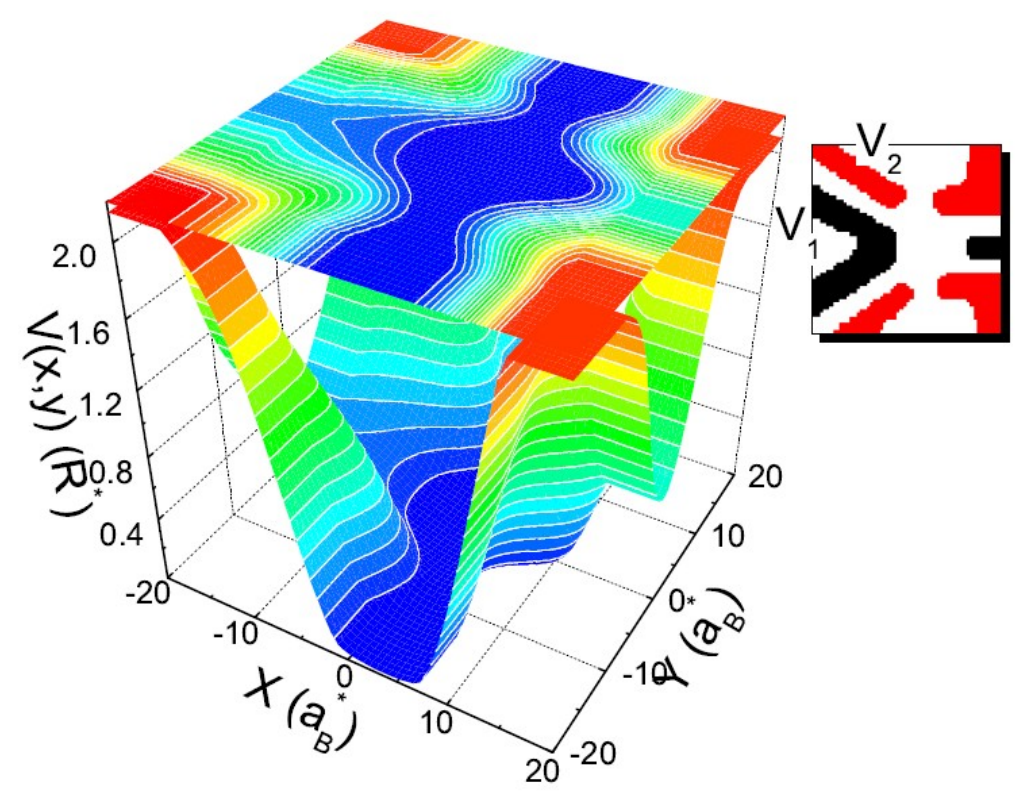

Figure 1. The self-consistent potential plotted in 3D as a function of the lateral coordinates. The lengths are in units of effective Bohr radius and energy is normalized with the effective Rydberg energy. The inset depicts the sample geometry, where S stands for source lead and D stands for the drain lead. Coupling of the QD to the leads is manipulated by changing the applied potential $V_{2}$.

\section{Results and Discussion}

The calculation of the electrostatic potential considering real sample geometries together with the electron-electron (e-e) interaction is a challenging issue. Since such a calculation cannot be done analytically for almost all the cases, usually numerical techniques are deployed. It is clear that for "more than a few" electron regime $(N>10)$ exact diagonalization methods are either impossible or very costly in terms of computational effort. It is favorable to use a mean field approximation to describe the (e-e) interactions, which is questionable in the "less than a few" electron regime. The commonly used approach to determine the bare confinement potential generated by the gates is the "frozen charge" approximation [12, which takes into account properly the gate pattern and the effect of the spacer between the gates and the 2DES. Since, it is not selfconsistent this approximation cannot account for the induced charges on the metallic gates defining the QD. The effects resulting from the induced charges and donor layer can be handled by solving the 3D Poisson equation self-consistently. Almost a decade ago M. Stopa introduced a very effective numerical scheme to describe the electrostatics of such samples [11, including the e-e interactions either using a full Hartree, i.e. solving the Poisson and Schrödinger equations self-consistently, or considering Thomas-Fermi approximation (TFA). The exchange-correlation interaction was accounted by a local density approximation (LDA) using the density functional theory (DFT). It was shown that the TFA is powerful enough to describe the electrostatic potential even if the 


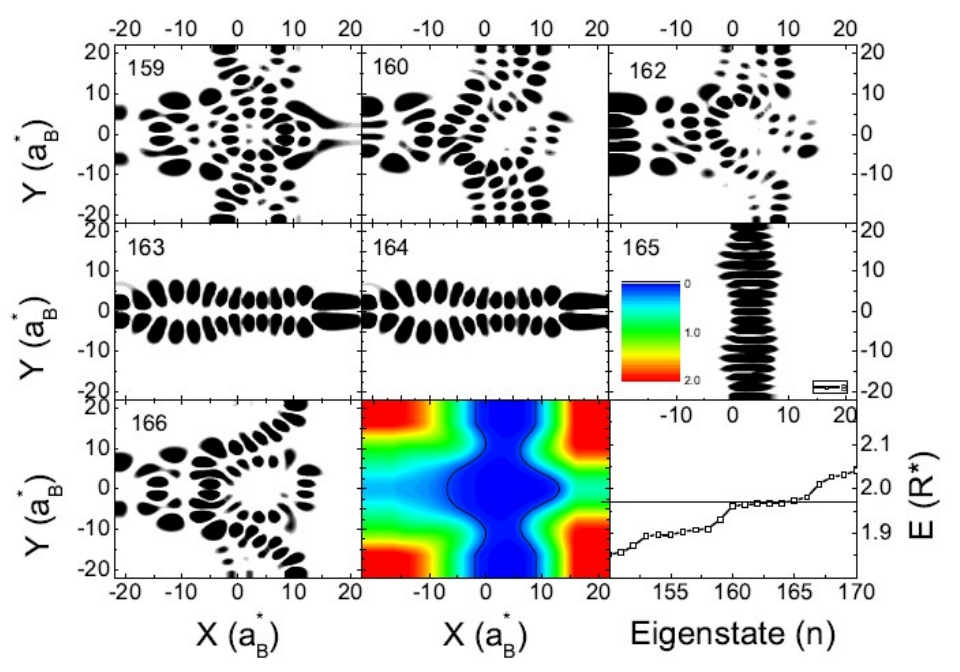

Figure 2. (a-g) Selected eigenfunctions residing at the $E=1.96$ plateau (indicated by the horizontal line in i) calculated for the color plotted potential (h), together with the energy spectrum near $E_{F}$. The state $=160$ present a slight asymmetry in coupling to source lead compared to the drain. The asymmetric potential distribution with in the dot is visible.

electrons are fully depleted in some regions of the sample [11].

Here, we stay in the TFA to calculate the electrostatic properties of the real sample geometry using the algorithm developed by A. Weichselbaum et. al [13, 14, which implements an efficient grid relaxation technique to solve the 3D Poisson equation. This approach was shown to be reliable to obtain the potential profiles in the "more than a few" electron regime considering QDs and quantum point contacts [10]. The next step in our calculation scheme is to obtain the single particle energies and states, which we do same as described in the previous section.

Figure. 1 presents the calculated potential profile for the sample geometry measured in Ref. [2]. We apply negative voltages to the gates shown in the inset. The upper and lower two gates (denoted by red areas) are kept at the same potential $V_{2}$, whereas the center gate (left black) and the plunger gate (right black) are biased with a fixed voltage $V_{1}$. Here, we consider a unit cell of $440 \times 440 \mathrm{~nm}^{2}$ spanned by $128 \times 128$ mesh matrix to calculate the self-consistent potential. The surface potential is fixed to $-0.75 \mathrm{~V}$ pinning the Fermi energy at the mid gap. The 2DES is some $100 \mathrm{~nm}$ below the surface followed by a thick GaAs layer. To achieve numerical convergence and satisfy the open boundary conditions 3 mesh points of dielectric material is assumed at all boundaries. In Figure. 1 fixed voltages of $V_{1}=-1.5 \mathrm{~V}$ and $V_{2}=-2.2 \mathrm{~V}$ are applied, the bulk electron density is estimated to be $3 \times 10^{11} \mathrm{~cm}^{-2}$ corresponding to $E_{F} \approx 12.75 \mathrm{meV}$, with the given density, the number of electrons in the dot $N$ is similar to 200. Figure. 2 presents the calculated single particle wave functions as a function of spatial coordinates, together with the 


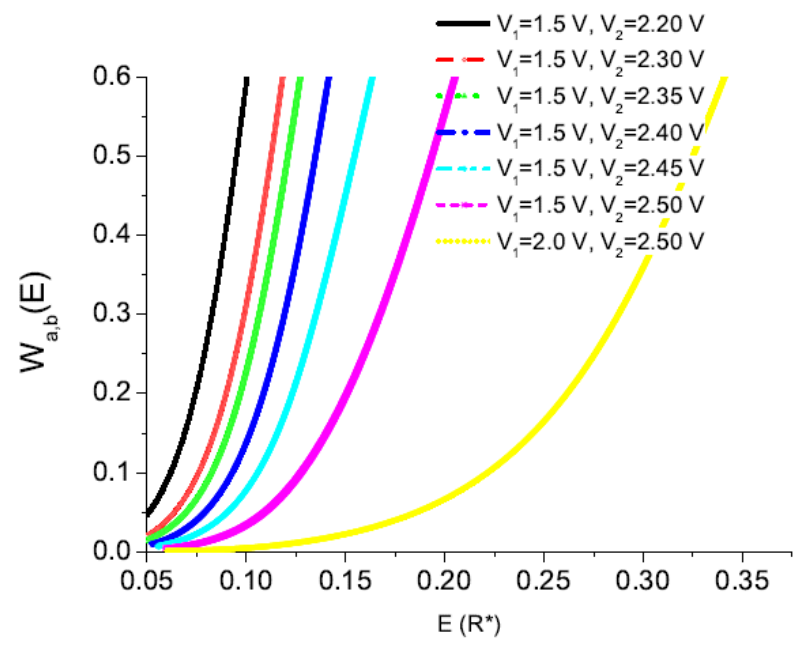

Figure 3. The transmission coefficients calculated at the barrier. The turning points are obtained from the self-consistent potential at the center of the barrier, where the energy of the incoming wave cuts along.

potential counter plot and the corresponding eigen energies versus the state number. We show the states residing at the energy plateau, which lay in the close vicinity of $E_{F}$ (depicted by the horizontal solid line in Figure. 2i). The states shown at the upper panel present the chaotic behavior, whereas the first two states of the mid panel are the non-propagating states. At $n=165$ a resonant channel is observed, meanwhile the highest state shown presents the chaotic behavior. These results show that, qualitatively, transport through state 165 is much probable compared to the others sitting at the same plateau. Although the single particle energy eigenvalues are close to each other a single channel is in charge of transport. At these gate voltages, the QD is loosely defined as one can see that it is possible to find an electron also at the left side of the actual QD. This situation is changed by applying a higher negative potential to the central and the plunger gates, $V_{1}=-2.0 \mathrm{~V}$. However, the QD potential is not rotationally symmetric even if one neglects the gates, since the center gate is geometrically different from the plunger gate. Now, we turn back our attention to the level width $\Gamma$ in a qualitative manner. As we have discussed $\Gamma$ becomes meaningful if one also considers both the source and drain leads. At this point it is useful to look at the transmission probability $W_{n}(a, b)(E)$, in Fig. 3 we show the this quantity as a function of energy of the incoming wave calculated within the WKB at various gate voltages, $V_{1}, V_{2}$. We see that, when the upper and the lower gates are biased with small potentials, the transmission increases linearly. This linearity changes if one applies higher voltages to the barriers, however, for higher energies the linearity is recovered. Such an observation leads us to conclude that, essentially the probability distribution determines the level widths, which may 
become asymmetric considering transport at different energies.

To summarize, we have calculated the self-consistent electrostatic potential exploiting the smooth variation of the bare potential within the TFA. Next we obtained the single particle eigenstates and energies considering a real sample geometry and crystal structure. We found, similar to the Ref. [4], that some single particle levels bunch and present a energy plateau while changing the state number. It was observed that, within these plateaus, not all the states contribute to the transport since the overlap of the dot wave functions and lead wave functions simply vanish. More interestingly, we found that at intermediately high energies, the wave functions are coupled to at least one of the leads much stronger than the ones in their close energy vicinity. This result, we believe, supports the phenomenological models, which attribute the abrupt change of the phase lapses to electron-electron interactions.

\section{Acknowledgments}

We would like to thank Jan von Delft for introducing us the "phase lapses" problem and motivating us to perform numerical calculations. Moty Heiblum is also acknowledged for his enlightening discussions. This work is partially supported by TUBiTAK, under grant no:109T083.

\section{References}

[1] A. Yacoby, M. Heiblum, D. Mahalu, and H. Shtrikman. Coherence and Phase Sensitive Measurements in a Quantum Dot. Physical Review Letters, 74:4047-4050, May 1995.

[2] M. Avinun-Kalish, M. Heiblum, O. Zarchin, D. Mahalu, and V. Umansky. Crossover from 'mesoscopic' to 'universal' phase for electron transmission in quantum dots. Nature, 436:529533, July 2005.

[3] G. Hackenbroich and H. A. Weidenmueller. Independent-Electron Model for the Phase of the Transmission Amplitude in Quantum Dots. ArXiv Condensed Matter e-prints:condmat/9502033, February 1995.

[4] P. G. Silvestrov and Y. Imry. Towards an Explanation of the Mesoscopic Double-Slit Experiment: A New Model for Charging of a Quantum Dot. Physical Review Letters, 85:2565-2568, September 2000.

[5] G. Hackenbroich. Phase coherent transmission through interacting mesoscopic systems. Physics Reports, 343:463-538, March 2001.

[6] C. Karrasch, T. Hecht, A. Weichselbaum, J. von Delft, Y. Oreg, and V. Meden. Phase lapses in transmission through interacting two-level quantum dots. New Journal of Physics, 9:123-+, May 2007.

[7] C. Karrasch, T. Hecht, A. Weichselbaum, Y. Oreg, J. von Delft, and V. Meden. Mesoscopic to Universal Crossover of the Transmission Phase of Multilevel Quantum Dots. Physical Review Letters, 98(18):186802-+, May 2007.

[8] P. G. Silvestrov and Y. Imry. Level-occupation switching of the quantum dot, and phase anomalies in mesoscopic interferometry. New Journal of Physics, 9:125-+, May 2007.

[9] A. Weichselbaum and S. E. Ulloa. Potential landscapes and induced charges near metallic islands in three dimensions. Phys. Rev E, 68(5):056707-+, November 2003.

[10] S. Arslan, E. Cicek, D. Eksi, S. Aktas, A. Weichselbaum, and A. Siddiki. Realistic modelling of 
quantum point contacts subject to high magnetic fields and with current bias at out of linear response regime. ArXiv e-prints, 803, March 2008.

[11] M. Stopa. Quantum dot self-consistent electronic structure and the Coulomb blockade. Phys. Rev. B, 54:13767-13783, November 1996.

[12] J. H. Davies, I. A. Larkin, and E. V. Sukhorukov. Modeling the patterned two-dimensional electron gas: Electrostatics. J. Appl. Phys., 77(9):4504, 1995.

[13] A. Weichselbaum and S. E. Ulloa. Potential landscapes and induced charges near metallic islands in three dimensions. Phys. Rev. E, 68(5):056707-+, November 2003.

[14] A. Weichselbaum and S. E. Ulloa. Tunability of qubit Coulomb interaction: Numerical analysis of top-gate depletion in two-dimensional electron systems. Phys. Rev. B, 74(8):085318-+, August 2006 . 